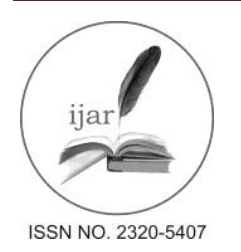

\section{Journal homepage:http://www.journalijar.com Journal DOI: 10.21474/IJAR01}

\section{RESEARCH ARTICLE}

INTERNATIONAL JOURNAL

OF ADVANCED RESEARCH

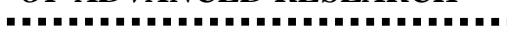

\title{
Experimental and Numerical Study of Gas-Liquid Flow for Gold Nanoparticles Synthesis.
}

*Sabrine Mejri, Jalila Sghaier and Ahmed Bellagi.

Unité Thermique et Thermodynamique des Procedes Industriels, Ecole Nationale d'ingenieurs de Monastir, Universite de Monastit, Rue Ibn El Jazzar, Monastir 5000, Tunisia.

\section{Manuscript Info}

\section{Manuscript History:}

Received: 15 May 2016

Final Accepted: 22 June 2016

Published Online: July 2016

Key words:

Photochemical reaction,

Gold nanoparticles,

Taylor flow,

T-junction microchannel

*Corresponding Author

\section{Abstract}

Gold nanoparticles, with a size ranging from 1 to $50 \mathrm{~nm}$, were synthesized in a microchannel reactor using a photochemical continuous two-phase flow method. Gold reduction was executed with a photoinitiator (Irgacure 2959) and by exposure to ultraviolet light. A numerical simulation, during which we used the Volume of Fluid method VOF in the software Ansys Fluent, was conducted as a preliminary study of the hydrodynamic behavior of the two phase flow (water-oxygen).

Sabrine Mejri. Copy Right, IJAR, 2016. All rights reserved:

\section{Introduction:-}

Gold colloid has been used for ages, from medical purposes and coloring glass in ancient history to many more applications in the modern world. Nanoparticles sizes ranging from $1 \mathrm{~nm}$ to $100 \mathrm{~nm}$ employed in diverse fields such as electronics, biology, medicine (MacMahon et al., 2008), data storage (Sun et al., 2000) etc. for they have such unique properties (Daniel et al., 2004), such as the optoelectronic and physiochemical properties, which are affected by the size and shape of the nanometals. For instance, for bio-sensing the size needed is ranging from 4 to $40 \mathrm{~nm}$ in diameter when $10 \mathrm{~nm}$ are needed for immunostaining (Rosi et al., 2005).Such biological and medical applications notably require high stability of nanoparticles in high ionic strength and physiological conditions while maintaining their physical and chemical properties.

Although with the emergence field of nanotechnology, the synthesis of nanoparticles with specific size and shape is of a huge importance, establishing a reproducible preparation method of prechosen size is difficult using the common chemical approaches. Turkevich proposed a simple method of synthesis in 1951 (Turkevich et al., 1951) which was refined later by Frens in 1970 (Frens et al., 1972). It consists essentially of producingmonodisperse spherical gold nanoparticles suspended in water of around $10-20 \mathrm{~nm}$ in diameter. Larger particles can be produced, but this comes at the cost of monodispersity and shape. It involves the reaction of small amounts of hot chloroauric acid with small amounts of sodium citrate solution. The colloidal gold will form because the citrate ions act as both a reducing agent and a capping agent. However, there are some disadvantages. The particles cannot be isolated from solution without irreversible aggregation which restricts their biological application. The citrate-stabilized AuNPs are vulnerable to aggregation at high ionic strength due to the change of the particle's surface charge. Therefore, the need for greater stability because of high sensitivity to the ionic strength and $\mathrm{pH}$ of the medium has driven researchers to explore alternative synthesis methods (Zhou et al., 2009).

Form chemical to green synthesis, researchers have devoted their efforts to develop methods of controlled synthesis of nanometals varying in sizes and in shapes (Sun et al., 2002). Sau et al. (2001) reported that controlling the size range of synthesized AuNPs by UV irradiation is an efficient, simple and fast technique with few control parameters. Experimental handling is easy and unlike the chemical reduction technique for seed preparation. Yang et 
al. (2007) introduced a continuous method for size and shape controlled synthesis of gold nanoplates and spherical nanoparticles (single crystals and polycrystals) using UV irradiation to initiate the reaction and PVP in solution which leads to the formation of spherical gold nanoparticles, and the size control could be realized by adjusting the PVP concentration. Tsunoyama et al. (2008) did successfully synthesize PVP stabilized gold clusters using a micromixer. They showed higher catalytic activity in ascorbic acid than those prepared in batch which concludes that reducing the cluster size is a mean to enhance the catalytic activity.

Continuous flow microreactors are able to improve the homogeneity of reactions and by extent the uniformity of the product (Jahn et al., 2008). Bollinger et al.(2006) reported that decreasing the channels diameter to the micrometer scale allows the easy change of experimental conditions within microseconds, as well as the reaction volume which will be greatly reduced for micrometers. An additional advantage of the continuous synthesis platforms is that numbering-up is simple and can be done by reactions taking place in parallel, thus shortening the development lab time to commercial production (Ying et al., 2008). Moreover, a continuous process is energy efficient due to the elimination of high energy consumption to repeat the heat and cooling in batch systems, and minimizes inventory of the overall process.

In this paper, we report a photochemical continuous flow method to synthesis monodisperse spherical gold nanoparticles, as well as a 2D simulation of a gas-liquid Taylor flow using the VOF method.

\section{Material and Methods:-}

Gold (III) Chloride Hydrate, Irgacure 2959 (1-[4-(2-Hydroxyethoxy)-phenyl]-2-hydroxy-2-methyl-1-propane-1one), PVP (Polyvinylpyrrolidone), were purchased from Sigma-Aldrich. The micro-reactor is a capillary with $1.0 \mathrm{~mm}$ inner diameter made of FEP Tubing (Fluorinated Ethylene Propylene) exposed to a UV lamp with wavelength irradiation ranging from 370 to $415 \mathrm{~nm}$. Distilled water was used in all the process.

Herein, our microreactor has $1 \mathrm{~mm}$ diameter and UV irradiation was introduced to initiate the photochemical reduction, where Irgacure 2959 is used as a photoinitiator, in a continuous segmented flow method. The flow in this procedure is a gas-liquid two phase flow composed of aqueous solutions, and Oxygen. We study the effect of dissolved gas concentration on gold reduction.

Table 1:- Experimental Conditions

\begin{tabular}{|l|l|l|}
\hline Solutions & Flow rates $(\mu \mathrm{L} / \mathrm{min})$ & Concentrations $(\mathrm{mM})$ \\
\hline $\mathrm{Au}$ & 50 & 0.165 \\
\hline Irgacure 2959 & 40 & 4 \\
\hline PVP & 20 & 20 \\
\hline
\end{tabular}

The solutions are delivered using separate syringe pumps through fluorinated ethylene propylene (FEP) capillaries (inner diameter: 1016um).Irgacure-2959 and $\mathrm{HAuCl} 4$ solution are encountered by a poly (ether-ether-ketone) (PEEK) Y-connector. Oxygen is delivered from an oxygen cylinder and its flow rate is regulated by a mass flow controller. 


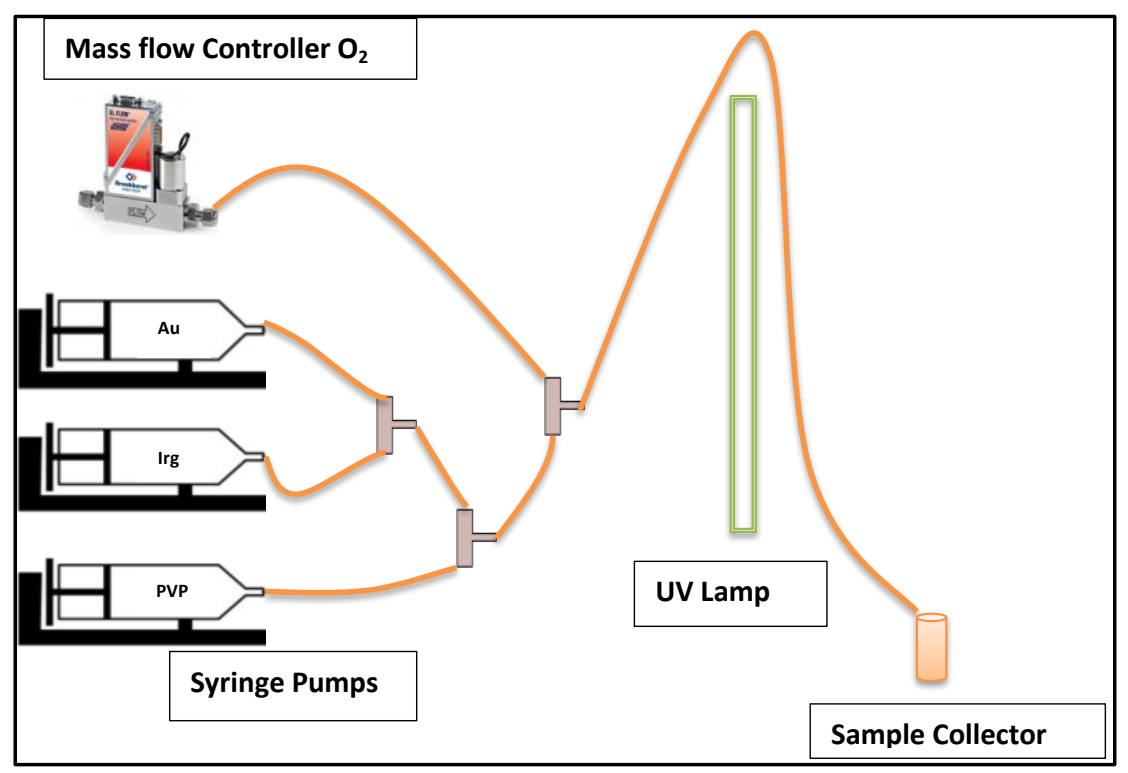

Figure 1:- Experimental Setup

Absorption measurements were done using a UV-Vis Jascospectrophotometer by placing adiluted sample in a quartz cuvette $(1 \mathrm{~cm}$ path length). The spectra were analyzed to determine the Plasmon absorption peak wavelengths and band widths unique to gold nanoparticles in order to determine the NPs diameters.

Transmission electron microscopy (TEM) was, as well, used to view the morphology and to determine the size of gold nanoparticles.

Numerically, using the VOF method of Ansys Fluent, we simulated a 2D unsteady water-oxygen (liquid-gas) flow through a T-junction capillary reactor. Our microreactor consists of two vertical mixing channels (one for water inlet and one for oxygen inlet) with $1 \mathrm{~mm}$ in diameter, as shown in figure 2.

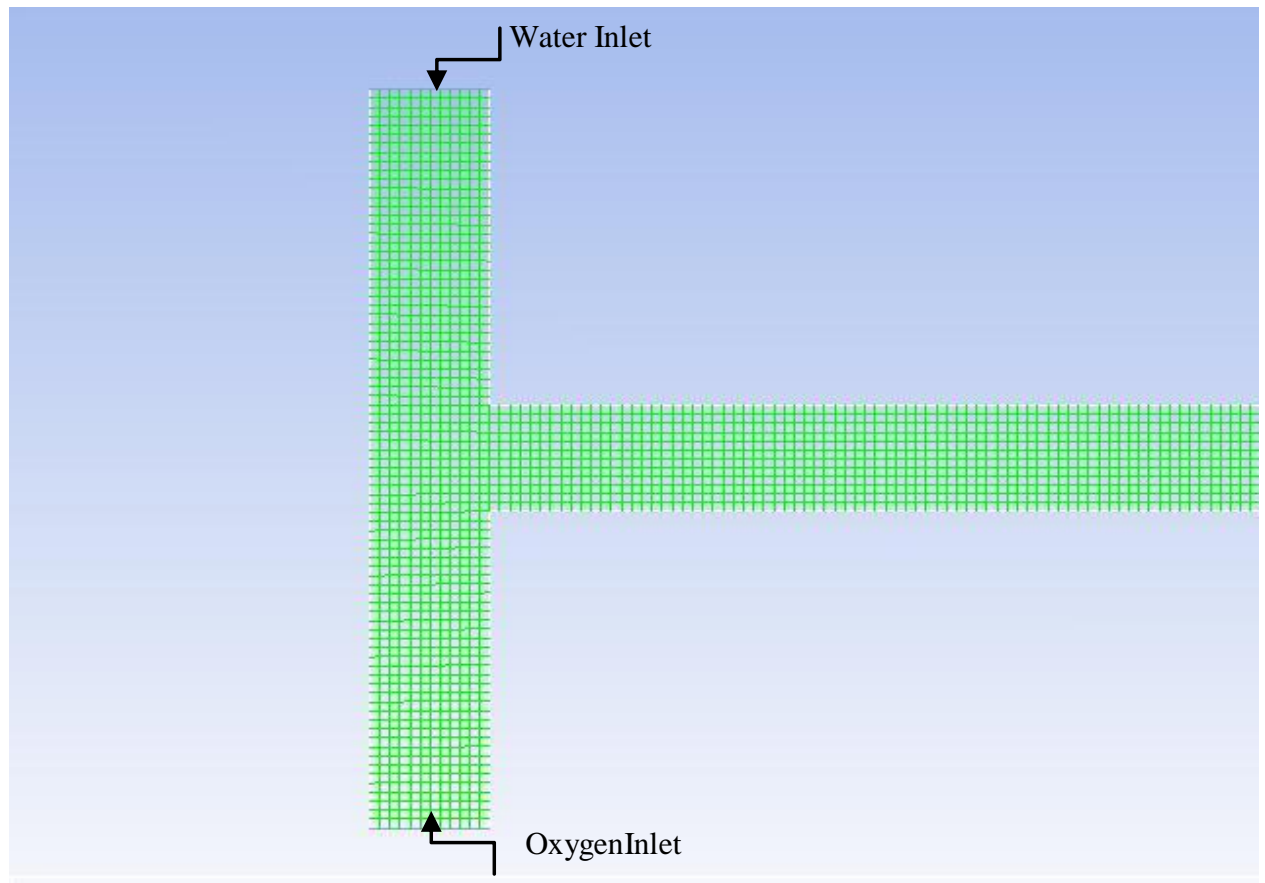

Figure 2:- Inlets of the Micro-channel. 
In our channel, the length to diameter ratio of $(\mathrm{L} / \mathrm{d}=100)$ is enough to ignore the entrance and exit effect. We generated 15108 cells using quadrilateral elements in Fluent meshing.

Firstly, our microreactor was filled with oxygen. Then, water and oxygen were fed from the two vertical mixing channels, respectively, at flow rates of $100 \mu \mathrm{l} / \mathrm{min}$ for water (density equals to $998.2 \mathrm{~kg} / \mathrm{m}^{\wedge} 3$ ) and $50 \mu \mathrm{l} / \mathrm{min}$ for oxygen (density equals to $1.292 \mathrm{~kg} / \mathrm{m}^{\wedge} 3$ ). The surface tension between water oxygen was set to $0.07 \mathrm{~N} / \mathrm{m}$. The flow is assumed to be unsteady and laminar. A no-slip boundary condition is imposed at the wall. computational fluid dynamics package, which utilizes the finite volume method for the spatial discretization. The VOF algorithm, which is a numerical technique which is used to track and locate the shape and position of the interface between two or more immiscible fluids, was implemented to model the Taylor flow through the microchannel reactor.

\section{Result and Discussion:-}

Gold nanoparticles (figure 3) were synthesized using a photochemicalcontinuous flow method. During the process of radical generation from the photoinitiatorIrgacure-2959, which would reduce gold ions into gold atoms and eventually to GNPs, the dissolved oxygen in the reaction media can play a significant role. Oxygen is trapped by the radical from irgacure-2959, which eventually produces carboxylic acid. While oxygen can influence the inherent reaction of radical generation, such carboxylic acid can also take part in stabilization of the incipient GNPs as capping agents onto the gold surface.

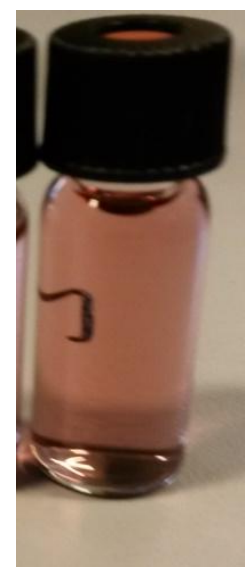

Figure. 3:- Sample of AuNPs solution

Figure 3 illustrates the AuNPs solutionwe collected. Using the UV-vis spectrophotometer, we obtained the absorbance spectrum of the solutions (Figure 4) which shows the absorbance curve. Haiss et al. (2007) established that it is possible to determine the size and concentration of GNPs directly from the UV-vis spectra data. This conclusion was based on a quantitative description of the absorbance of GNPs in the size range from 5 to $100 \mathrm{~nm}$ of the mean free path corrected Mie theory which was found in good agreement with experimental data. Although the Haiss equations were valid, the UV-vis spectroscopy is not the most precise way to determine size of AuNPs.TEM observations (figure 5), and the study of the size distribution (figure 6), show that forour sample the size ranges from 1 to $50 \mathrm{~nm}$. 


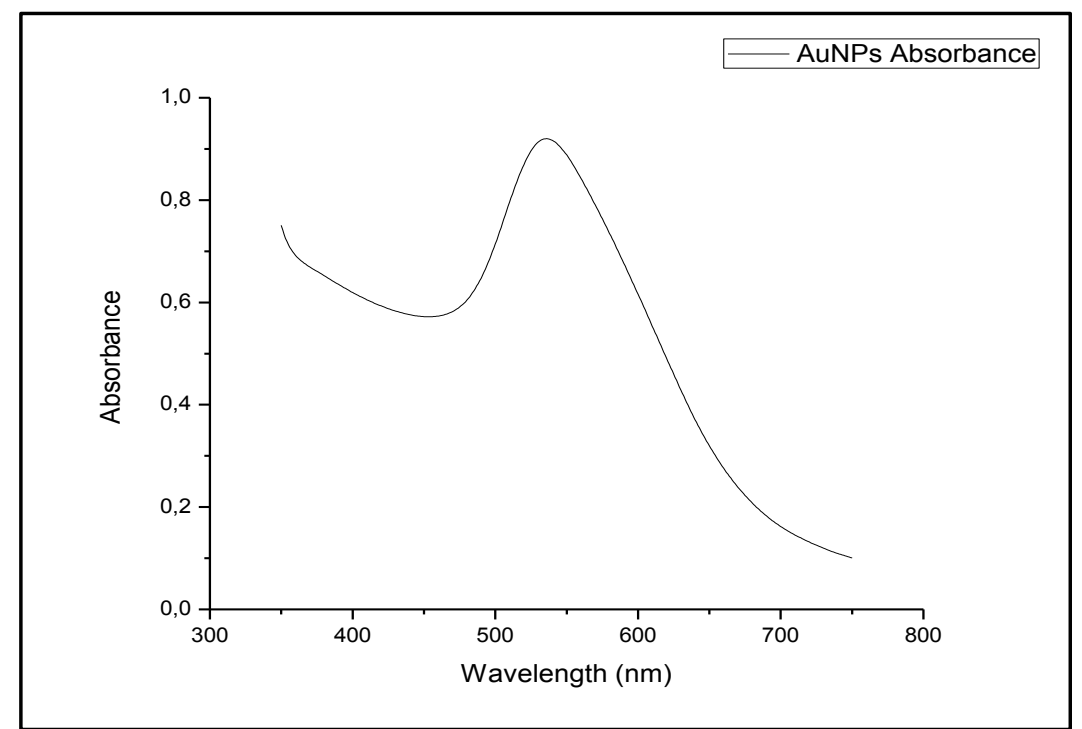

Figure 4:- Absorbance Spectrum of AuNPs obtained

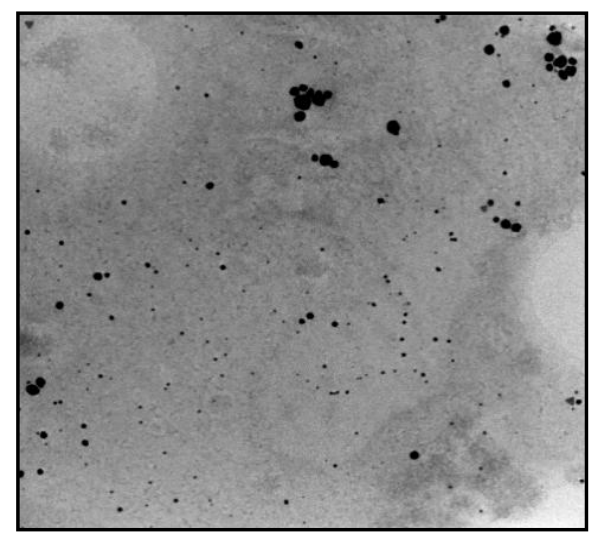

Figure 5:- TEM observation of AuNPs

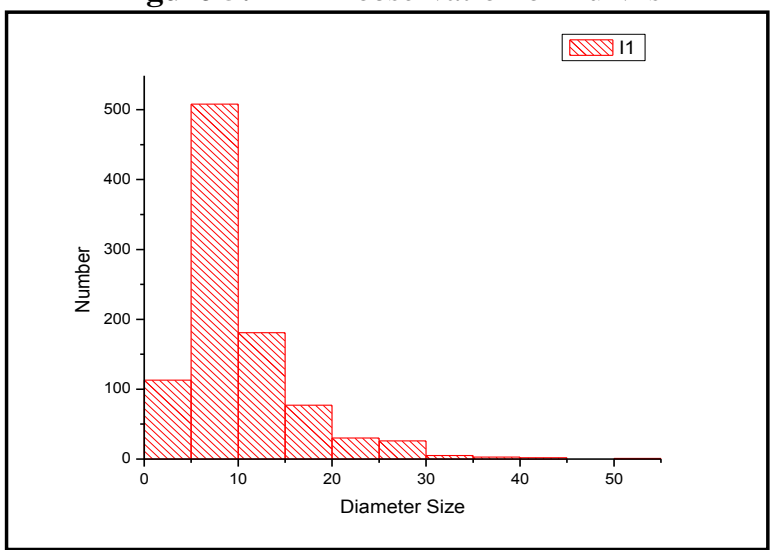

Figure 6:- Size distribution of our sample

However, as gold can easily deposit on the microreactor channel (figure 7), we opt to use later on an inert liquid component as a lubricating layer between aqueous phase and wall. 


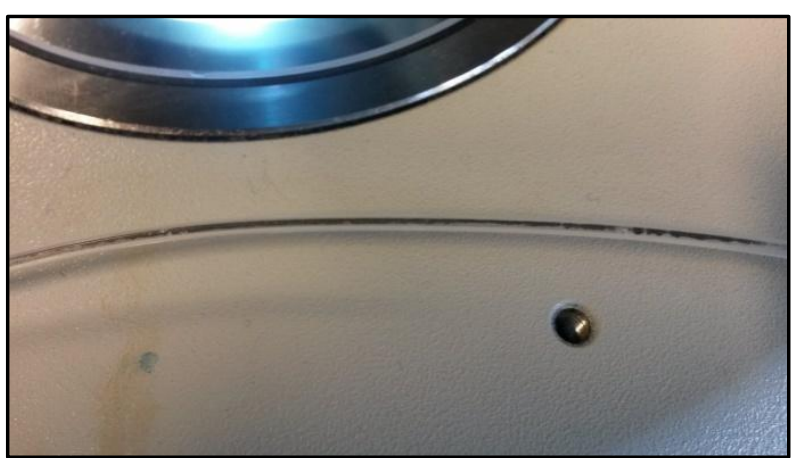

Figure 7:- Gold deposition on the inner surface of the capillary

The hydrodynamic behavior of the two-phase (water-oxygen) flow throughthe T-junction microchannelwas investigated numerically. The 2-D simulations were performed with fixed flow rates at the water and oxygen inlets. Figure 8 shows contour plot ofthe density of the two fluids inside the microchannel.It is in good match with Figure 9 , showing the flow from the experiments.

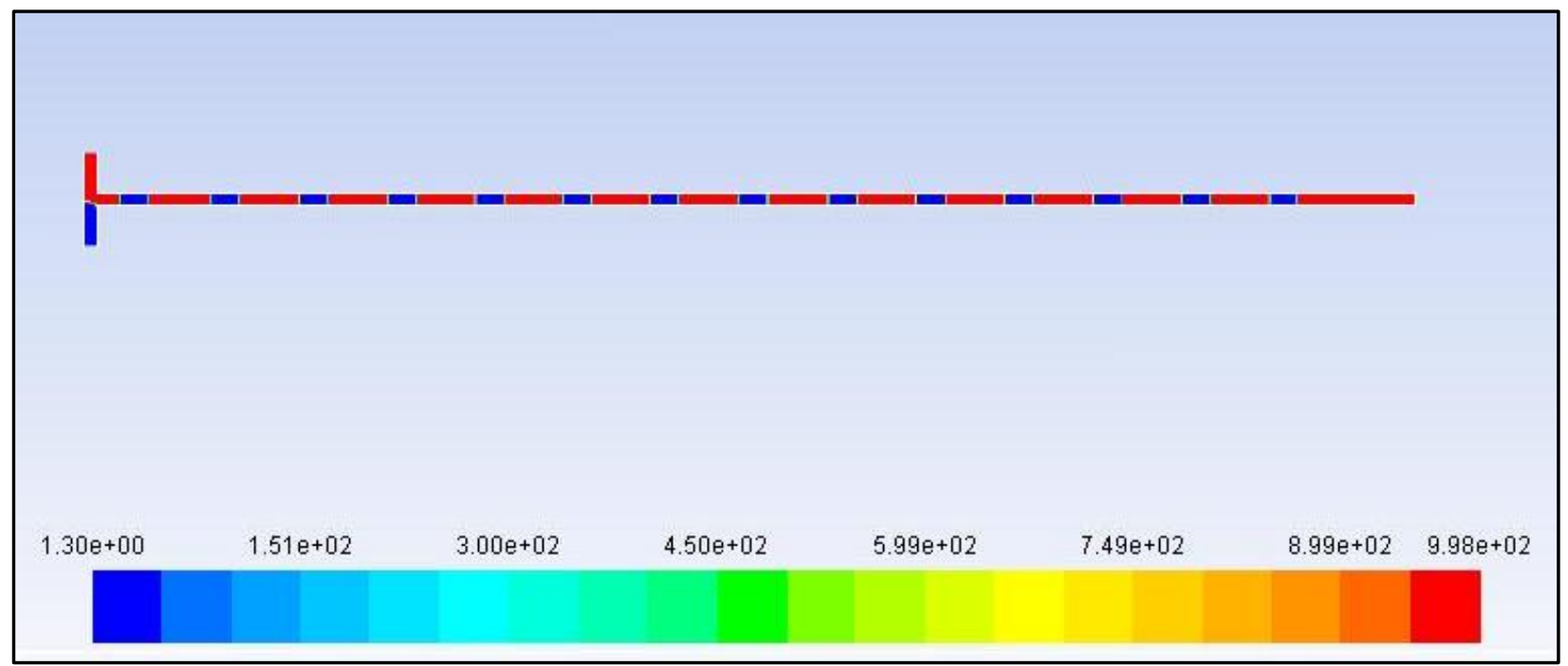

Figure 8:- Density mixture in the T-junction microchannel at 3s

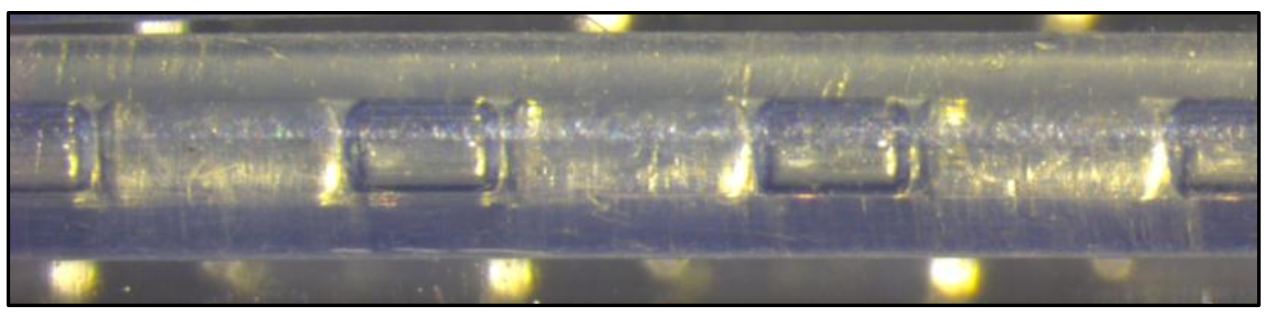

Figure 9:- Hydrodynamic behavior of the two-phase flow in the microchannel

\section{Conclusion:-}

We were successful in reducing gold chloride hydrate into nanoparticles, not exceeding $50 \mathrm{~nm}$ in size. Our method involved a continuous two-phase flow (gas-liquid) in a microchannel reactor with a T-junction mixing zone. A part of the microreactor was exposed to UV light with wavelength ranging from 370 to $415 \mathrm{~nm}$. Although this photochemical method was a success, we report a serious deposition problem on the inner surface of the capillary, which can reduce the length of the microchannel exposed and by extension the residence time in the microreactor.

To solve this problem we will introduce a third more viscous phase into the capillary to avoid the contact between the gold solution and the wall of the microchannel.

The preliminary numerical study shows matching to the experiments. This will allow us to further our hydrodynamic investigation to determine the pressure drop, the gas holdup along with the flow quality. 


\section{Acknowledgment:-}

We would like to acknowledge help provided by Professor EvgenyRebrov, director of the Energy Intensified Reactor Engineering Laboratory, School of Engineering, University of Warwick, and the access to his laboratory. Advice and Assistance of Dr. NikolayCherkasov, member of the same lab, have been of a great value to make this work.

\section{References}

1. S. J. MacMahon, M. H. Mendenhall, S. Jain, F. Currell. (2008). Radiotherapy in the presence of contrast agent: a general figure of merit and its application to gold nanoparticles, Physics in Medicine and biology, Vol 53 Number 20.

2. S. Sun, C.B. Murray, D. Weller, L. Folks, A.Moser. (2000). MonodispersedFePt nanoparticles and ferromagneticFePtnanocrystalsuperlattices, Science, Volume 287, Issue 5460: 1989-1992

3. M.C. Daniel, D. Astruc. (2004). Gold nanoparticles: Assembly, supramolecular chemistry, quantum-sizerelated properties, and applications toward biology, catalysis and nanotechnology, Chemical Reviews, 104 (1): 293-346.

4. N.L. Rosi, C.A. Mirkin. (2005). Nanostructures in biodiagnostics, Chemical Reviews, 105 (4): 1547-1562.

5. J. Turkevich, P. C. Stevenson, J. Hillier. (1951). A study of the nucleation and growth processes in the synthesis of colloidal gold, Discuss. Faraday. Soc. 11, 55-75

6. G. Frens. Particle size and sol stability in metal colloids, Colloid \& Polymer Science, 1972, 250, 736-741

7. M. Zhou, B. Wang, Z.N. Rozynek, Z. Xie, J.O. Fpssum, X. Yu, S. Raaen. (2009). Minute synthesis of extremely stable gold nanoparticles, Nanotechnology, 20 (50)

8. Y. Sun, Y. Xia. Shape-controlled synthesis of gold and silver nanoparticles, Science 2002, 298, Issue 5: 21762179.

9. Tapan K. Sau, Anjali Pal, N.R. Jana, Z.L. Wang,Tarasankar Pal. (2001).Size controlled synthesis of gold nanoparticles using photochemically prepared seed particles, 5; Journal of Nanoparticle Research 3: 257-261

10. S. Yang, T. Zhang, L. Zhang, S. Wang, Z. Yang, B. Ding. (2007). Continuous synthesis of gold nanoparticles and nanoplates with controlled size and shape under UV irradiation; Colloids and Surfaces A: Physicochem. Eng. Aspects 296: 37-44.

11. H. Tsunoyama, N. Ichikuni, T. Tsukuda.(2008).Microfluidic Synthesis and Catalytic Application of PVPStabilized, 1 nm Gold Clusters,; Langmuir, 24, 11327-11330.

12. A. Jhan, J.E. Reiner, W.N Vreeland, M. Gaitan. (2008). Preparation of nanoparticles by continuous-flow microfluidics, J. Nanopart.Res, 10: 925-934

13. J. Boleininger, A. Henkel, V. Reuss, C. Sönnichsen. (2006). Microfluidic continuous flow synthesis of rodshaped gold and silver nanocrystals, Phys. Chem. Chem. Phys. 8: 3824-3827

14. Y. Ying, G. Chen, Y. Zhao, Q. Yuan. (2008). A high throughput methodology for continuous preparation of monodispersed nanocrystals in microfluidic reactors, The Chemical Engineering Journal 135(3):209-215

15. W. Haiss, N. T. K. Thanh, J. Aveyard, D. G. Fernig. (2007). Determination of Size and Concentration of GoldNanoparticles from UV - Vis Spectra, Analytical Chemistry, Vol. 79, No. 11: 4215 - 4221 